\title{
Fermion Field on Random-Block Lattice
}

\author{
T. W. Chiu ${ }^{\text {a * }}$
}

a Physics Department, National Taiwan University, Taipei, Taiwan 106, R.O.C.

The massless fermion field interacting with an external gauge field is studied on 2D random-block lattice. The fermion propagator, the gauge invariant currents and their divergences, and current-current correlations are computed. They are in good agreement with the continuum field theory.

\section{INTRODUCTION}

The doubling problem of lattice fermion can be understood from the viewpoint of functional integral measure. It has been proposed that the fermion doubling is only an artifact of the deficiency of a single lattice, and can be evaded by incorporating a proper measure provided by an ensemble of random-block lattices (RBL) [1]. For free fermion fields on $2 \mathrm{D}$ and $4 \mathrm{D} \mathrm{RBL}$, all ensemble averaged path integrals [1-3] are free of fermion doubling, and in good agreement with the continuum field theory. In this talk, recent results of massless fermion field interacting with a background gauge field on 2D RBL are reported. The fermion propagator, the gauge invariant currents and current-current correlations are computed. They all agree with the continuum values. The divergence of the axial-vector current yields the same anomaly in continuum field theory. These results support the proposal that RBL could provide a proper measure for evaluating functional integrals involving fermion fields.

A random-block lattice (RBL) [1] in D dimensional Euclidean space is constructed by cartesian product of $D$ sets of random coordinates, one from each dimension. Each set of random coordinates are presumably randomly distributed in an interval $[0, L]$ with uniform probability, where $L$ is the spatial size of the lattice in each dimension. The separation between any two neighbouring coordinates in each dimension thus obeys the Poisson distribution. This property is essential

*Research supported by National Science Council, R.O.C. under the grant NSC82-0208-M002-013. for a random lattice to provide a proper measure for evaluating the functional integrals involving fermion fields. An example of a 2-dimensional RBL is shown in Fig. 1

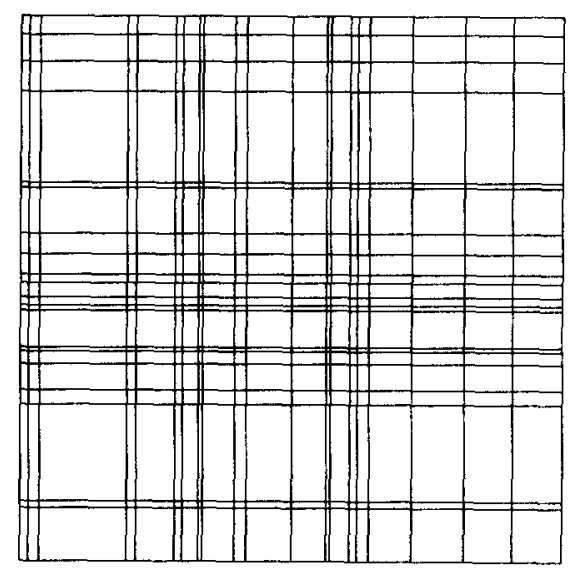

Figure 1. An example of 2D random-block lattice.

\section{FERMION PROPAGATOR}

The action of a massless fermion field interacting with an external gauge field on 2D RBL can be written as

$A_{L}=\sum_{i, \mu} \omega_{i} K_{i}^{\mu} \bar{\psi}_{i} \gamma_{\mu}\left[U_{\mu}(i) \psi_{i+\mu}-U_{\mu}^{\dagger}(i-\mu) \psi_{i-\mu}\right]$

where $\psi_{i}$ and $\bar{\psi}_{i}$ are two independent 2 component spinors at site $i, \omega_{i}=\prod_{\mu} \frac{\left(x_{i+\mu}-x_{i-\mu}\right)}{2}$ is the weight at site $i, K_{i}^{\mu}=\left(x_{i+\mu}-x_{i-\mu}\right)^{-1}$ is 
the inverse of the distance between the sites $i+\mu$ and $i-\mu, U_{\mu}(i)=\exp \left[i \int_{x_{i}}^{x_{i+\mu}} A_{\nu}(x) d l_{\nu}\right]$ is the link variable pointing from the site $i$ to the site $i+\mu$, and the gamma matrices are chosen to be $\gamma_{1}=\sigma_{1}, \gamma_{2}=\sigma_{2}$ and $\gamma_{5}=-i \gamma_{1} \gamma_{2}=\sigma_{3}$ where $\sigma_{i}, i=1,2,3$ are Pauli matrices. The fermion propagator from the site $\mathrm{y}$ to the site $\mathrm{x}$ is

$$
\begin{aligned}
S_{F}(x, y) & =\frac{1}{V^{N-2}} \int \prod_{i=1}^{N-2} d^{2} z_{i} S_{F}^{L}(x, y) \\
& \equiv \gamma_{\mu} S_{\mu}(x, y)
\end{aligned}
$$

where $V$ is the volume of the lattice, $N$ is the total number of sites of the lattice and the integration over all positions of the $N-2$ sites is defined as summing over all possible randomblock lattices generated with two fixed sites corresponding to the end-points of the propagator, and $S_{F}^{L}(x, y)$ is the fermion propagator on a given RBL. In the following, the gauge field is chosen to be $A_{\mu}(x)=\delta_{\mu, 1} A_{0} \sin \left(\omega x_{2}\right)$ with $\omega=\frac{2 \pi}{L}$ and $A_{0}=0.1$. The fermion propagator $S_{F}^{L}(x, y)$ on a given RBL is proportional to the probability amplitude of the transition from $\mathrm{y}$ to $\mathrm{x}$ on this particular lattice, which is the sum of the probability amplitude over all possible paths between $y$ and $x$. In continuum, many other paths are possible. In order to take into account other paths which are allowed in the continuum, many different RBL must be generated while holding $x$ and $y$ fixed, and the propagator in each RBL is evaluated. Since each RBL is equally probable, the resulting propagator is then the arithmetic average of the propagators over an ensemble of RBL. The real parts and imaginary part$\mathrm{s}$ of the ensemble-averaged fermion propagator $S_{F}(x, y)=\gamma_{\mu} S_{\mu}(x, y)$ over $2048 \mathrm{RBL}$, each having $20 \times 20$ sites, are plotted in Fig. 2. One of the end-points of the propagator is fixed at the origin $y=(0,0)$, while the other end-point is along the diagonal of the lattice $\left(x_{2}=x_{1}\right)$. Anti-periodic boundary conditions have been imposed. It is evident that the ensemble-averaged propagators agree very well with the continuum results ( the solid lines). On the other hand the fermion propagator on a regular lattice vanishes for both endpoints $\mathrm{x}$ and $\mathrm{y}$ lying on the diagonal of the lattice.
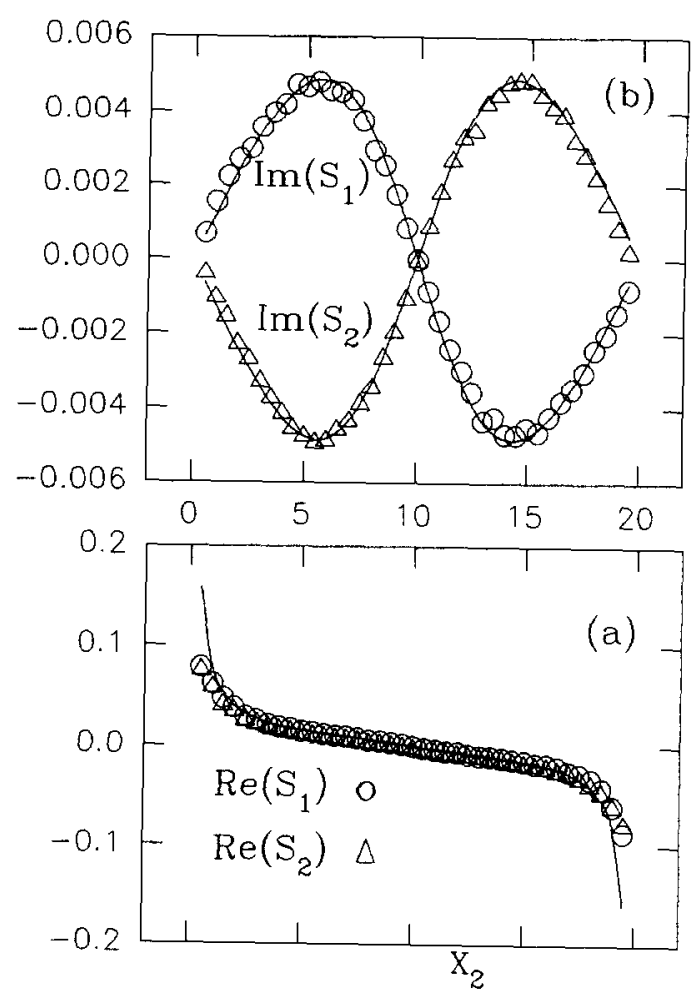

Figure 2. The massless fermion propagator in an external sinusoidal gauge field. (a) Real part$\mathrm{s}$ of $S_{1}(x, y)$ and $S_{2}(x, y)$. (b) Imaginary parts of $S_{1}(x, y)$ and $S_{2}(x, y)$.

\section{GAUGE INVARIANT CURRENTS}

One of the most serious difficulties of the fermion field on a single lattice is the absence of chiral charge due to the complete cancellation of chiral charges among the primary mode and the doubled modes. Locally, this is equivalent to the absence of the divergence of axial vector curren$t$, the anomalies which govern various important physical quantities, in particular the decay of the neutral pion to photons. It is therefore important to investigate the behavior of the axial vector current and see whether it behaves properly after ensemble averaging over a set of RBL. In continumm, the axial vector current is not well defined and can be regulated using gauge invariant point-splitting [5]. In general, we consider the 
gauge invariant point-splitting current for any operator $\Gamma$ in the spinor space,

$$
\begin{aligned}
& j_{\Gamma}(x, \epsilon)=\frac{1}{2} \bar{\psi}\left(x-\frac{\epsilon}{2}\right) \Gamma \psi\left(x+\frac{\epsilon}{2}\right) \times \\
& \exp \left[i \int_{x-\frac{\epsilon}{2}}^{x+\frac{\epsilon}{2}} A_{\nu}(x) d l_{\nu}\right]+(\epsilon \leftrightarrow-\epsilon)
\end{aligned}
$$

which is invariant under the $U_{V}(1)$ gauge transformation. It can be shown that the divergence of the vacuum expectation value of the axial vector current $\left(\Gamma=\gamma_{\mu} \gamma_{5}\right)$ is [4]

$\lim _{\epsilon \rightarrow 0} \partial_{\mu}<j_{\mu}^{5}(x, \epsilon)>=-\frac{\epsilon_{\mu \nu} F_{\mu \nu}}{2 \pi}$.

Using RBL for regularization, the vacuum expectation value of the gauge invariant point-splitting current is

$\left\langle j_{\Gamma}(x, \epsilon)>=\frac{1}{V^{N-2}} \int \prod_{i=1}^{N-2} d^{2} z_{i}<j_{\Gamma}^{L}(x, \epsilon)>\right.$

where $\left\langle j_{\Gamma}^{L}(x, \epsilon)>\right.$ is the vacuum expectation value of the gauge invariant point-splitting current on a RBL. The vector current $\left\langle j_{\mu}(x, \epsilon)\right\rangle$ and the axial vector current $\left\langle j_{\mu}^{5}(x, \epsilon)>\right.$ are measured using $4096 \mathrm{RBL}$, each having $20 \times 20$ sites, along the diagonal ( $\left.x_{2}=x_{1}\right)$, with point-splitting $\epsilon=(a, a)$, where $\mathrm{a}$ is the average lattice spacing. They both agree very well with the continuum values [6]. The divergence of the vector current $\partial_{\mu}\left\langle j_{\mu}(x, \epsilon)>\right.$ and the divergence of the axial vector current $\partial_{\mu}<j_{\mu}^{5}(x, \epsilon)>$ along the diagonal are evaluated by numerical differentiation [6] and are plotted in Fig. 3. The divergence of the vector current ( denoted by circles ) vanishes at any positon and therefore agrees with the continuum field theory. It also implies that the gauge symmetry is maintained on RBL. The divergence of the axial vector current (denoted by triangles) agrees with the result in continuum field theory ( the solid line )

$-\frac{\epsilon_{\mu \nu} F_{\mu \nu}}{2 \pi}=A_{0} \omega \cos \left(\omega x_{2}\right) / \pi$.

\section{DISCUSSIONS}

It has been shown that RBL can provide a proper measure for functional integrals involving

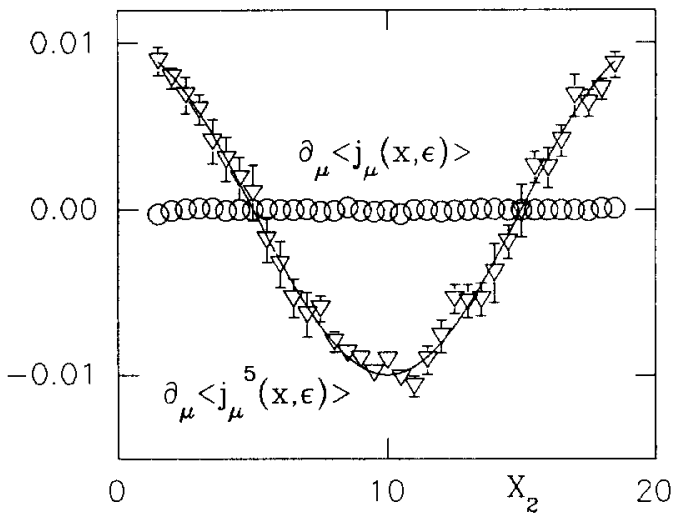

Figure 3. The divergence of axial vector current and vector current

a massless fermion field interacting with an external gauge field. The fermion propagator, the gauge invariant currents and current-current correlations [6] are all in good agreement with the continuum values. The divergence of the axialvector current yields the same anomaly in continuum field theory. Since the axial anomaly is only due to the one-loop quantum corrections, it is expected that the same must hold for a chiral fermion interacting with a dynamical gauge field. The gauge invariant composite boson propagators and the vacuum polarization tensor are also free of doublings [6]. Since the whole scheme of RBL regularization does not rely on the dimensionality of the theory, it is expected that the same will hold for a chiral fermion field interacting with a gauge field in four dimensions.

\section{REFERENCES}

1. T.W. Chiu, Phys. Lett. B 206 (1988) 510.

2. T.W. Chiu, Phys. Lett. B 217 (1989) 151.

3. T.W. Chiu, "Field Theory on the RandomBlock Lattice ", Proceedings of the 25th International Conference on High Energy Physics, p.1149. ( World Scientific, 1991 ).

4. J. Schwinger, Phys. Rev. 128 (1962) 2425.

5. J. Schwinger, Phys. Rev. 82 (1951) 664.

6. T.W. Chiu, " Massless Fermion Field on 2D Random-Block Lattice", NTUTH-93-16, September, 1993. 